\title{
A goal programming technique for railroad passenger scheduling
}

\author{
Masoud Yaghini $^{\mathrm{a}^{*}}$, Alireza Alimohammadian ${ }^{\mathrm{a}}$, and Samaneh Sharifi ${ }^{\mathrm{b}}$
}

${ }^{a}$ Department of Railroad Transportation, Iran University of Science \& Technology, Tehran, Iran ${ }^{b}$ Department of Industrial Engineering, KNTU University of Technology, Tehran, Iran

\begin{tabular}{l}
\hline A R T I C L E I N F O \\
\hline Article history: \\
Received October 1, 2011 \\
Received in Revised form \\
October, 3, 2011 \\
Accepted 15 December 2011 \\
Available online \\
17 December 2011 \\
\hline Keywords: \\
Goal programming \\
Mixed Integer Programming \\
Railroad planning \\
Passenger scheduling
\end{tabular}

\begin{abstract}
A B S T R A C T
Railroad industry has received tremendous challenges in the world in terms of handling cost and efficiency. For many years, the railroad business lost money in many countries such as Japan until many governments decided to privatize the industry in an attempt to reduce the cost components and to increase the efficiency of various units, significantly. In this paper, we propose a new goal programming technique to handle two objectives of operating cost and the number of direct passengers travel by train. We consider different types of trains for public transportation of passengers in order to make the proposed model of this paper more realistic. The implementation of the proposed model is demonstrated using some numerical examples to show the effectiveness of the method.
\end{abstract}

\section{Introduction}

Managing the cost of transportation is one of the most challenging issues in passenger scheduling especially in developing countries (Barnhart \& Laporte, 2007). This is a relatively big industry and it is responsible to transport millions of people between various cities each year. The business is often highly regulated and it is not an easy task to increase fares since it can easily hike inflation. In some countries like Japan, when both private and public sectors were involved for managing the industry, the private one was profitable but the public one was not. However, when the government privatized the public unit, in less than ten years, the privatized transportation unit became as one of the profitable and well-known units listed in S\&P index. The moral story was that management of this industry plays an essential role for increasing the productivity of this industry. Kroeger (2005) in his book discussed different issues surrounding this industry in terms of political and public negotiation issues.

During the past few decades, there have been tremendous changes in world's railroad industry in terms of providing efficient methods for increasing the productivity and the efficiency. Presently,

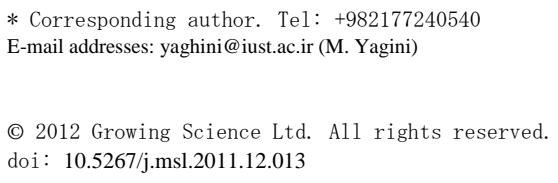


many people prefer train to other facilities because train is one of the most secure facilities in the world. During the past few decades, there have been significant attempts to provide efficient methods for cost management of train scheduling. Suryani et al. (2010) presented forecasting techniques to provide reliable estimate on demand for terminal capacity expansion using a system dynamic methodology. They reported some important issues influencing demand estimation such as population, growth domestic product, level of service impact, etc. Bussieck et al. (1996) proposed another demand estimation for runway and passenger terminal capacity expansion to meet future demand using system dynamic methodology. Tsai et al. (2009) presented neural network based temporal feature models for short-term railway passenger demand forecasting. Goossens et al. (2004, 2006) presented a line planning model for solving line planning problems in which lines could have different halting patterns. Brønmo et al. (2010) presented some mathematical model for ship scheduling problem with flexible cargo sizes. The well-known pickup and delivery problem with time window is normally formulated as large scale optimization problem and solving such problem is often time consuming. They presented Dantzig-Wolfe procedure to handle the difficulties arising to this problem. Liu and Kozan (2009) presented a method for scheduling trains as a blocking parallelmachine job shop scheduling problem and applied their method for a real-world case study in Queensland railroad industry.

D'Ariano et al. (2007) studied a train scheduling problem with railway infrastructure managers when they are in real-time traffic control. Once train operations are faced with uncertainty, a new conflictfree timetable of feasible arrival and departure times needs to be re-computed so that we can minimize the deviation from the original one. The problem can be stated as a big job shop problem with no-store constraints. They estimated time separation among trains, and modeled the scheduling problem with an alternative graph formulation. They also developed a branch and bound method, which enhances implication rules enabling to increase the computation. They applied their method on a bottleneck area of the Dutch rail network and explained that a truncated version of the method could give optimal or near optimal solutions in less computational time. Peeters and Kroon (2008) proposed a model and a branch-and-price technique to determine an efficient railway rolling stock circulation on a set of train lines. Lindner (2000) and Scholl (2005) in their Phd work presented a comprehensive study on railroad scheduling problems using different techniques such as branch-andprice method. Ghoseiri and Ghannadpour (2010) presented a hybrid genetic algorithm to solve a multi-depot homogenous locomotive assignment problem with time windows. They first introduced a mathematical model using vehicle routing problem with time windows (VRPTW) and then they used a cluster-first, route-second approach to change the multi-depot locomotive assignment into a set of single depot problems and provided the solutions for each problem, independently.

They performed some experimental results with a set of 15 completely random generated instance problems and reported some encouraging results. Chung et al. (2009) presented a hybrid genetic algorithm for train sequencing in the Korean railway similar to what Ghoseiri and Ghannadpour did. There are also several works to use heuristic methods for solving railways problems (Lee \& Chen, 2009). There are also several works, which use Neural network to solve railway problems. Tsai et al. (2009), for instance used Neural network based temporal feature models for short-term railway passenger demand forecasting. Another alternative solution to determine the capacity of train scheduling is to use system dynamic methods (Suryani et al., 2010). Bussieck et al. (1996) presented fuzzy theory for train scheduling problems. Claessens et al. (1998) used location techniques for cost allocation of railroad problems. Burdett and Kozan (2009) presented various techniques for inserting additional trains into existing timetables.

Liu and Kuzan (2009) considered the train scheduling problem as a blocking parallel-machine job shop scheduling (BPMJSS) problem. In their modeling formulation, all trains, single-track sections and multiple-track segments, respectively, are synonymous with jobs, single and parallel machines, and an operation was considered as the movement/traversal of a particular train across a section. They 
solved a parallel-machine job-shop-scheduling (PMJSS) problem using an improved shifting bottleneck procedure (SBP) method without considering blocking conditions for a real-world application from Queensland Rail and analyzed the results. The results indicated that their proposed methodology would be a very useful tool for the real-life train scheduling problems. Mesquita and Paias (2008) used set partitioning/covering-based methods for the integrated vehicle and crew scheduling problem. They presented an algorithm with two stages, which starts with a pre-processing phase to define the set of tasks and to reach an initial set of duties. In a second phase, they used a linear programming relaxation of the models using a column generation scheme and used branch-andbound whenever the resulting solution does not provide integer solution.

The proposed study of this paper presents a goal programming technique to determine the optimal allocation of train scheduling problem with the minimization of the operating cost and maximization of the number of direct travelled passengers. There passengers travel from an origin to destination with no change in train. The proposed study first presents the problem statement in section 2 . Section 3 explains the results and finally concluding remarks are given in the last to summarize the contribution of the paper.

\section{Problem statement}

The following notations are used for the proposed model of this paper.

\section{Counters}

e

$r$

$h$

$a, b$

\section{Sets}

V

$E$

$H$

$R$

\section{Parameters}

$d_{a, b}$

$u(e)$

$t_{r}^{h}$

$\gamma^{h}$

$C x_{r}^{h}$

BO

$W_{1}$

$w_{2}$

$c_{h}$

$d(e)$

$T$

$p^{h}$

$C z_{r}^{h}$

$M$

$s_{a, b}$ counter of the arcs in network $e \in E$

Counter of lines $r \in R$

Counter for the type of train

Counters associated with the origin and destination of station $a, b \in V$

Sets of all stations

Sets of all arcs

Sets of all types of train

Sets of all suggested lines

Demand for trip from origin a to destination $b$

Upper bound for the number of permitted trip on arc $e$

Time required for trip of type $h$ for line $r$

Efficiency ratio of train type $h$

Variable cost associated with train type $h$ traveling to line $r$

Objective cost associated with operating expenses

Weight for total fixed and variable costs

Weight for direct travelled passengers

The capacity of train type $h$

Total demand for arc $e$

Total time available for scheduling trains

Number of train of type $h$

Fixed cost associated with train type $h$ in route $r$

A big number

A set of arcs for the shortest distance between $a$ and $b$ 
Decision variables

$y_{r, a, b}^{h}$

$z_{r}^{h}$

$x_{r}^{h}$

$s^{+}$

$s^{-}$
An integer variable determines number passengers directly traveled from station $a$ to $b$ by line $r$ and train type $h$

A binary variable, which specifies the existence of train type $h$ in line $r$

An integer variable indicating the number of trips on line $r$ by train type $h$

Slack variable

Surplus variable

The proposed goal programming model of this paper is formulated as follows.

$$
\begin{array}{ll}
\min \left\{s_{1}^{-}, s_{2}^{+}, s_{2}^{-}\right\} & \\
\sum_{r \in R} \sum_{h \in H}\left(c x_{r}^{h} x_{r}^{h}+c z_{r}^{h} z_{r}^{h}\right)+s_{1}^{+}-s_{1}^{-}=B o & \\
\sum_{a, b \in V} \sum_{e \in r \mid r \in R_{a, b}} \sum_{h \in H} y_{r, a, b}^{h}+s_{2}^{+}-s_{2}^{-}=\sum_{a, b \in V} d_{a, b} & \\
\sum_{e \in r \mid r \in R_{a, b}} \sum_{h \in H} y_{r, a, b}^{h} \leq d_{a, b} & \forall a, b \in V \\
\sum_{e \in r \mid r \in R_{a, b}} y_{r, a, b}^{h} \leq c_{h} x_{r}^{h} & \forall r \in R, e \in r, h \in H \\
\sum_{e \in r \mid r \in R_{a, b}} \sum_{h \in H} x_{r}^{h} \leq u(e) & \forall e \in E \\
\sum_{e \in r \mid r \in R_{a, b}} \sum_{h \in H} c_{h} x_{r}^{h} \geq d(e) & \forall e \in E \\
\sum_{r \in R} \frac{2 t_{r}^{h} x_{r}^{h}}{T \gamma^{h} \leq p^{h}} & \forall h \in H \\
x_{r}^{h} \leq M z_{r}^{h} & \forall r \in R, h \in H \\
s_{1}^{+} \times s_{1}^{-}=0 \& s_{2}^{+} \times s_{2}^{-}=0 & \\
x_{r}^{h} \in Z^{+}, y_{r, a, b}^{h} \in Z^{+}, z_{r}^{h} \in\{0,1\}, s^{+} \geq 0, s^{-} \geq 0 & \forall r \in R, \forall h \in H, \forall a, b \in V
\end{array}
$$

The objective function in Eq. (1) arranges that minimum amount of the objective function occurs on the desired level and tries to move as many direct passengers as it is possible based in Eq. (3). In practice the problem consists of two objective functions, which are given in constrains 2 and 3. In Eq. (2), the lower cost is more desirable, slack variable can get positive value but surplus variable has to have minimum value. On the other hand, in Eq. (3) we look to have more direct passengers from origin to destination. Therefore, it is undesirable not to meet demand and it is also undesirable to more too many semi empty trains because it will cost more. Therefore, we consider both slack and surplus variables and they could be in terms of Eq. (12) or Eq. (13) as follows,

$$
\min \left(s_{1}^{-}+s_{2}^{+}+s_{2}^{-}\right),
$$

or

$\min \left(w_{1} s_{1}^{-}+w_{2} s_{2}^{+}+w_{2} s_{2}^{-}\right)$. 
According to Eq. (12), we are looking for maximum transportation of direct passengers and when the relative importance of the two objective functions is different, we can switch to Eq. (13). Note that Eq. (13) is reduced to Eq. (12) when have $w_{1}=w_{2}$. According Eq. (4), total number of direct passengers who travel using all types of train between origin and destination does not have to exceed total demand. Eq. (5) specifies that we can transfer all passengers proportion to available resources in terms of different types of trains. In Eq. (7) $d(e)$ is calculated based on Eq. (14) as follows,

$$
d(e)=\sum_{a, b \in V \mid e \in s_{a, b}} d_{a, b} \quad \forall e \in E
$$

Eq. (8) determines the number of trains and Eq. (9) assigns a positive value for the number of trips on line $r$ by train type $h$ only when the structure of problem confirms that this move should happen. Eq. (10) explains that only one variable of slack or surplus must maintain a positive value, simultaneously and finally, Eq. (11) specifies the type of variables.

\section{A Numerical example}

Consider an example of railway transportation with seven nodes shown in Fig. 1.

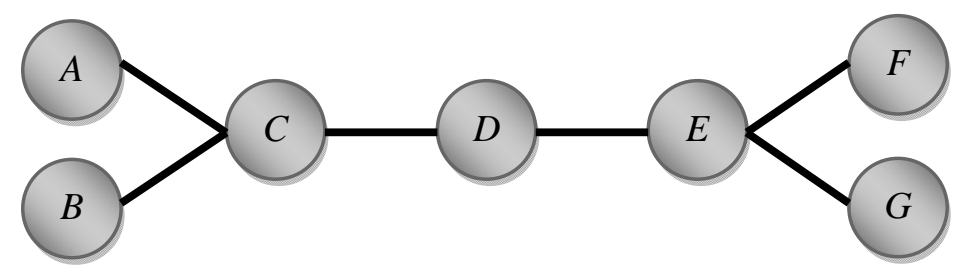

Fig. 1. Sample graph for numerical example

As we can observe from the example, there are seven stations and 6 arcs for the proposed case study of this numerical example. In this example, we consider two types of trains, which are shown with type 1 and type 2. The time, cost and other input data are given in Table 1 as follows,

\section{Table 1}

Origin, destination, time, fixed and variable costs associated with each line and each type of train

\begin{tabular}{lcccccccc}
\hline & & \multicolumn{4}{c}{ Traveling time } & Fixed cost & \multicolumn{3}{c}{ Variable cost } \\
\hline Line & Origin & Destination & Type 1 & Type 2 & Type 1 & Type 2 & Type 1 & Type 2 \\
\hline 1 & A & B & 0.9 & 1.1 & 4 & 3 & 0.45 & 0.55 \\
2 & A & C & 0.4 & 0.5 & 2 & 1.5 & 0.2 & 0.25 \\
3 & A & D & 1 & 1.2 & 4 & 3 & 0.5 & 0.6 \\
4 & A & E & 1.4 & 1.6 & 6 & 4.5 & 0.7 & 0.8 \\
5 & A & F & 2.1 & 2.4 & 8 & 6 & 1.05 & 1.2 \\
6 & A & G & 2.2 & 2.4 & 8 & 6 & 1.1 & 1.2 \\
7 & B & C & 0.5 & 0.6 & 2 & 1.5 & 0.25 & 0.3 \\
8 & B & D & 1.1 & 1.2 & 4 & 3 & 0.55 & 0.6 \\
9 & B & E & 1.5 & 1.7 & 6 & 4.5 & 0.75 & 0.85 \\
10 & B & F & 2.2 & 2.7 & 8 & 6 & 1.1 & 1.35 \\
11 & B & G & 1.9 & 2.1 & 8 & 6 & 0.95 & 1.05 \\
12 & C & D & 0.6 & 0.7 & 2 & 1.5 & 0.3 & 0.35 \\
13 & C & F & 1 & 1.1 & 4 & 3 & 0.5 & 0.55 \\
14 & C & F & 1.7 & 1.9 & 6 & 4.5 & 0.85 & 0.95 \\
15 & C & G & 1.4 & 1.5 & 6 & 4.5 & 0.7 & 0.75 \\
16 & D & E & 0.4 & 0.45 & 2 & 1.5 & 0.2 & 0.23 \\
17 & D & F & 1.1 & 1.2 & 4 & 3 & 0.55 & 0.60 \\
18 & D & G & 0.8 & 0.85 & 4 & 3 & 0.4 & 0.43 \\
19 & E & F & 0.7 & 0.8 & 2 & 1.5 & 0.35 & 0.40 \\
20 & E & G & 0.4 & 0.45 & 2 & 1.5 & 0.2 & 0.23 \\
21 & F & G & 1.1 & 1.2 & 4 & 3 & 0.55 & 0.60 \\
\hline
\end{tabular}


As we have explained, there two types of train in our example. There are 25 trains of type 1 with the capacity of 100 passengers, total time of 20 hours and $\gamma^{h}=0.6$. There are also 30 trains associated with train type 2, the capacity of each train is 150 passenger and we have 30 trains available with $\gamma^{h}=0.5$. Table 2 shows details of demand between each origin and destination.

\section{Table 2}

Demand for between each station

\begin{tabular}{|c|c|c|c|c|c|c|c|}
\hline Stations & A & $\mathrm{B}$ & $\mathrm{C}$ & $\mathrm{D}$ & $\mathrm{E}$ & $\mathrm{F}$ & $G$ \\
\hline A & & 1000 & 1500 & 900 & 750 & 600 & 550 \\
\hline B & & & 1300 & 1100 & 850 & 700 & 750 \\
\hline $\mathrm{C}$ & & & & 1600 & 1400 & 1200 & 1100 \\
\hline $\mathrm{D}$ & & & & & 1000 & 600 & 800 \\
\hline $\mathrm{E}$ & & & & & & 1200 & 1300 \\
\hline $\mathrm{F}$ & & & & & & & 950 \\
\hline
\end{tabular}

The upper bounds for arcs $A C, B C, C D, D E, E F$ and $E G$ are 60, 75, 105, 105, 75 and 60, respectively. In addition the value of the objective cost is 170 . We have solved the proposed model of this paper using Lingo 8 software package. Table 3 summarizes efficient values of the implementation of the proposed model for different weights. As we can observe, the weights of $w_{1}$ and $w_{2}$ play important role on the final solution.

\section{Table 3}

Efficient solution for different values of $w_{1}$ and $w_{2}$

\begin{tabular}{ccccccc}
\hline$w_{1}=0.66$ & $w_{2}=0.33$ & $w_{1}=0.50$ & $w_{2}=0.50$ & $w_{1}=0.33$ & $w_{2}=0.66$ & OF \\
\hline Type 1 & Type 2 & Type 1 & Type 2 & Type 1 & Type 2 & Line \\
\hline 0 & 7 & 0 & 7 & 0 & 17 & 1 \\
0 & 0 & 1 & 0 & 0 & 0 & 2 \\
0 & 0 & 0 & 8 & 0 & 0 & 3 \\
19 & 6 & 2 & 3 & 11 & 4 & 4 \\
0 & 17 & 0 & 4 & 2 & 0 & 5 \\
0 & 4 & 0 & 16 & 8 & 14 & 6 \\
0 & 0 & 0 & 0 & 0 & 9 & 7 \\
8 & 0 & 0 & 0 & 0 & 2 & 8 \\
0 & 0 & 0 & 9 & 0 & 0 & 9 \\
0 & 8 & 0 & 11 & 0 & 8 & 10 \\
0 & 21 & 17 & 0 & 0 & 8 & 11 \\
0 & 5 & 0 & 0 & 0 & 10 & 12 \\
0 & 0 & 0 & 0 & 0 & 0 & 13 \\
0 & 0 & 0 & 13 & 24 & 0 & 14 \\
0 & 0 & 0 & 0 & 0 & 2 & 15 \\
0 & 0 & 0 & 0 & 0 & 0 & 16 \\
0 & 0 & 0 & 0 & 0 & 0 & 17 \\
0 & 0 & 0 & 0 & 0 & 0 & 18 \\
0 & 0 & 0 & 0 & 0 & 0 & 19 \\
1 & 0 & 0 & 2 & 0 & 0 & 20 \\
0 & 10 & 0 & 7 & 0 & 7 & 21 \\
\hline
\end{tabular}


As we can observe, when we consider different weights, the final solutions are changes. For instance, when we choose $w_{1}=0.33, w_{2}=0.66,17$ trains type 2 are assigned to line 1 and 11 trains type 1 are assigned to line 4 . There are also 14 type 2 trains to line 6 and 8 trains type 1 to the same line.

\section{Conclusion}

In this paper, we have proposed a new goal programming technique to handle two objectives of operating expenditures and the number of direct passengers travel by various types of trains. The proposed model of this paper has been formulated as a mixed integer programming technique. One advantage of the proposed model is that we can consider different types of trains in terms of train capacity, cost components, etc. This makes our problem formulation more realistic since in reality, customer may prefer to pay more to receive faster and more reliable services. The implementation of the proposed model has been demonstrated using some numerical examples to show the effectiveness of the method.

\section{Acknowledgment}

The authors would like to thank the anonymous referees for the constructive comments on earlier version of this work.

\section{References}

Barnhart, C. \& Laporte, G. (2007). Handbook in OR \& MS. Vol. 14, Elsevier B.V.

Bussieck, M. R., Kreuzer, P., \& Zimmermann, U. T. (1996). Optimal lines for railway systems. European Journal of Operational Research, 96, 54-63.

Brønmo, G., Nygreen, B., \& Lysgaard, J. (2010). Column generation approaches to ship scheduling with flexible cargo sizes. European Journal of Operational Research, 200(1), 139-150.

Burdett, R. L., \& Kozan, E. (2009). Techniques for inserting additional trains into existing timetables. Transportation Research, Methodological, 43(8-9), 821-836.

Claessens, M. T., van Dijk, N. M., \& Zwaneveld, P. J. (1998). Cost optimal allocation of passenger lines. European Journal of Operational Research, 110, 474-489.

Chung, J., Moo, S., \& Choi, I. (2009). A hybrid genetic algorithm for train sequencing in the Korean railway. Omega, 37(3), 55-65.

D’Ariano, A., Pacciarelli, D., \& Pranzo, M. (2007). A branch and bound algorithm for scheduling trains in a railway network. European Journal of Operational Research, 183(2), 643-657.

Ghoseiri, K., \& Ghannadpour, F. (2010). A hybrid genetic algorithm for multi-depot homogenous locomotive assignment with time windows. Applied Soft Computing, 10(1), 53-65.

Goossens, J. W., Hoesel, S. V., \& Kroon, L. (2006). On solving multi-type railway line planning problem. European Journal of Operational Research, 168(2), 403-424.

Goossens, J. H. M., van Hoesel, C. P. M., \& Kroon, L. G. (2004). A branch-and-cut approach for solving railway line-planning problems. Transportation Science, 38, 379-393.

Kroeger, A. (2005). Retiring the Crow Rate: A Narrative of Political Management. University of Alberta Press, 280.

Lindner, T. ( 2000). Train schedule optimization in public rail transport. PhD thesis, TU Braunschweig.

Liu, S.Q., \& Kozan, E. (2009). Scheduling trains as a blocking parallel-machine job shop scheduling problem. Computers \& Operations Research, 36(10), 2840-2852.

Lee, Y., \& Chen, C. (2009). A heuristic for the train pathing and timetabling problem. Transportation Research, Methodological, 43(8-9), 837-851.

Mesquita, M., \& Paias, A. (2008). Set partitioning/covering-based approaches for the integrated vehicle and crew scheduling problem. Computers \& Operations Research, 35(5) 1562-1575.

Peeters, M., \& Kroon, L. (2008). Circulation of railway rolling stock: a branch-and-price approach. Computers \& Operations Research, 35(2), 538-556. 
Scholl, S. (2005). Customer-oriented line planning. PhD thesis, University of Kaiserslautern.

Suryani, E., Chou, S., \& Chen, C. H. (2010). Air passenger demand forecasting and passenger terminal capacity expansion: A system dynamics framework. Expert Systems with Applications, 37(3), 2324-2339.

Tsai, T. H., Lee, C. K., \& Wei, C. H. (2009). Neural network based temporal feature models for short-term railway passenger demand forecasting. Expert Systems with Applications, 36(2), 37283736. 